\title{
HELICAL PLATING - A NOVEL TECHNIQUE TO INCREASE STIFFNESS IN DEFECT FRACTURES
}

\author{
M. Lenz ${ }^{1,2}$, P. Varga ${ }^{1}$, D. Mischler ${ }^{1}$, B. Gueorguiev ${ }^{1, *}, K$ Klos $^{3}$, A. Fernandez dell'Oca ${ }^{4}$, P. Regazzoni ${ }^{5}$, \\ R.G. Richards ${ }^{1}$ and S.M. Perren ${ }^{1,5}$ \\ ${ }^{1} \mathrm{AO}$ Research Institute Davos, Davos, Switzerland \\ ${ }^{2}$ Department of Trauma, Hand and Reconstructive Surgery, University Hospital Jena, Jena, Germany \\ ${ }^{3}$ Gelenkzentrum Rhein-Main, Hochheim, Germany \\ ${ }^{4}$ Department of Traumatology, Hospital Britanico, Montevideo, Uruguay \\ ${ }^{5}$ AO Foundation, Davos, Switzerland
}

\begin{abstract}
Single-plate fixation bridging bone defects provokes nonunion and risks plate-fatigue failure due to underdimensioned implants. Adding a helical plate to bridge the fracture increases stiffness and balances load sharing. This study compares the stiffness and plate surface strain of different constructs in a transverse contact and gap femoral shaft fracture model.

Eight groups of six synthetic femora each were formed: intact femora; intact femora with lateral locking plate; contact and gap transverse shaft osteotomies each with lateral locking plate, lateral locking plate and helical locking plate, and long proximal femoral nail. Constructs underwent non-destructive quasi-static axial and torsional loading. Plate surface strain evaluation was performed under $200 \mathrm{~N}$ axial loading.

Constructs with both lateral and helical plates demonstrated similar axial and torsional stiffness independent of the contact or gap situations - being significantly higher compared to lateral plating $(p<0.01)$. Torsional stiffness of the constructs, with both lateral and helical plates in the gap situation, was significantly higher compared to this situation stabilised by a nail $(p<0.01)$. Plate surface strain dropped from $0.3 \%$ in the gap situation with a lateral plate to $<0.1 \%$ in this situation with both a lateral and a helical plate.

Additional helical plating increases axial and torsional construct stiffness in synthetic bone and seems to provide well-balanced load sharing. Its use should be considered in very demanding situations for gap or defect fractures, where single-plate osteosynthesis provides inadequate stiffness for fracture healing and induces nonunion.
\end{abstract}

Keywords: Double plating, helical plate, locking plate, surface strain, nonunion, fatigue failure.

*Address for correspondence: Prof. Boyko Gueorguiev, PhD, AO Research Institute Davos, Clavadelerstrasse 8, 7270 Davos Platz, Switzerland.

Telephone number: +41 786656674 Email: boyko.gueorguiev@aofoundation.org

Copyright policy: This article is distributed in accordance with Creative Commons Attribution Licence (http://creativecommons.org/licenses/by-sa/4.0/).

\section{Introduction}

The stiffness and strength provided by plate osteosynthesis of long-bone fractures enable rapid functional recovery of the limb. When the reduced fragments build a solid continuous bridge through contact, the fractured bone can share the load, thus protecting the plate from fatigue failure (Perren et al., 2014). In defect fractures or fractures with reduced bone quality, load sharing through the bone is not possible and plate failure occurs (Fig. 1a) (Perren et al., 2014). Axial load is the main load component in lower extremity fractures. Using a central load carrier reduces the bending moment in femoral shaft defect fractures when compared to lateral plating (Liporace and Yoon, 2019). Wherever possible, intramedullary nailing of these fractures is the current golden standard. Depending on fracture localisation and pre-existing implants, lateral locked plating must be performed. A series of distal periprosthetic femoral fractures, fixed with a lateral locked plate, reveal a failure rate of up to $26 \%$ (Beeres et al., 2020; Ebraheim et al., 2012; Hoffmann et al., 2012). If an interfragmentary contact cannot be established or is lost later on, addition of a second plate may protect the primary implant from fatigue failure (Fig. 1b) (Perren et al., 2014).

In a fractured bone allowing interfragmentary mobility, e.g. in comminuted fractures bridged by an implant acting as a splint, bone healing occurs mainly 
by callus formation (Fig. 1b) (Comiskey et al., 2010; Elkins et al., 2016; Perren, 2002). Locked-plate fixation bridging a fracture gap evokes a three-dimensional fracture motion. Whereas appropriate magnitude of longitudinal motion is presumed to stimulate fracture healing, shear motion is suspected to inhibit it and to induce nonunion (Elkins et al., 2016). The amount of shear motion at the fracture site depends on different parameters. It disproportionally increases relative to the longitudinal motion with increasing plate working length (Elkins et al., 2016). Compared to the respective stainless steel plates, the softer titanium plates with short working lengths allow a greater amount of longitudinal motion (Elkins et al., 2016; Rodriguez et al., 2016). Stiffer constructs are more likely to result in healing complications (Rodriguez et al., 2016). Large strains have the risk of resulting in fatigue failure if healing is retarded, while low strains do potentially provide insufficient healing stimulus and can lead to delayed union or nonunion (Rodriguez et al., 2016). Large interfragmentary motion and the corresponding repeated high implant stress can induce fatigue failure (Holzman et al., 2016).

Double plating reduces the overall motion at the fracture site and protects from fatigue implant failure (Todorov et al., 2018). Different options for double plating at the femur exist. A second plate could be placed orthogonally to the lateral plate (Bottlang et al., 2015; Perren et al., 2014) in shaft fractures or at the medial condyle in distal femoral fractures (Sanders et al., 1991; Steinberg et al., 2017). The essential advantage of the helical plate is that it can be applied minimally invasive, and its application is simple with limited surgical trauma (Beeres et al., 2020; Fernandez Dell'Oca, 2002a; Fernandez Dell'Oca, 2002b; Perren et al., 2014). The function of a helical plate consists mainly in increasing stiffness, resulting in lower strain at same load and in exonerating a part of the load on the lateral plate (Perren et al., 2014). When choosing a sufficient length, and only fixing the helical plate at its very proximal and distal ends, secondary fracture healing occurs in the clinical situation; whereas single lateral plating can lead to nonunion and plate breakage (Fig. 1a,c) (Beeres et al., 2020; Perren et al., 2014). This study aimed to compare construct stiffness of a single lateral locked plate fixation without and with additional helical plate application (double-plate fixation) and an intramedullary nail fixation (as the golden standard) in a simple transverse femoral shaft fracture construct with interfragmentary contact and gap, respectively. The secondary aim was to analyse the effect of adding the helical plate on the lateral plate surface strain. It was hypothesised that adding a helical plate would reduce surface strain on the lateral plate and increase construct stiffness, especially in fractures where the fragments were not in contact but separated by a gap.
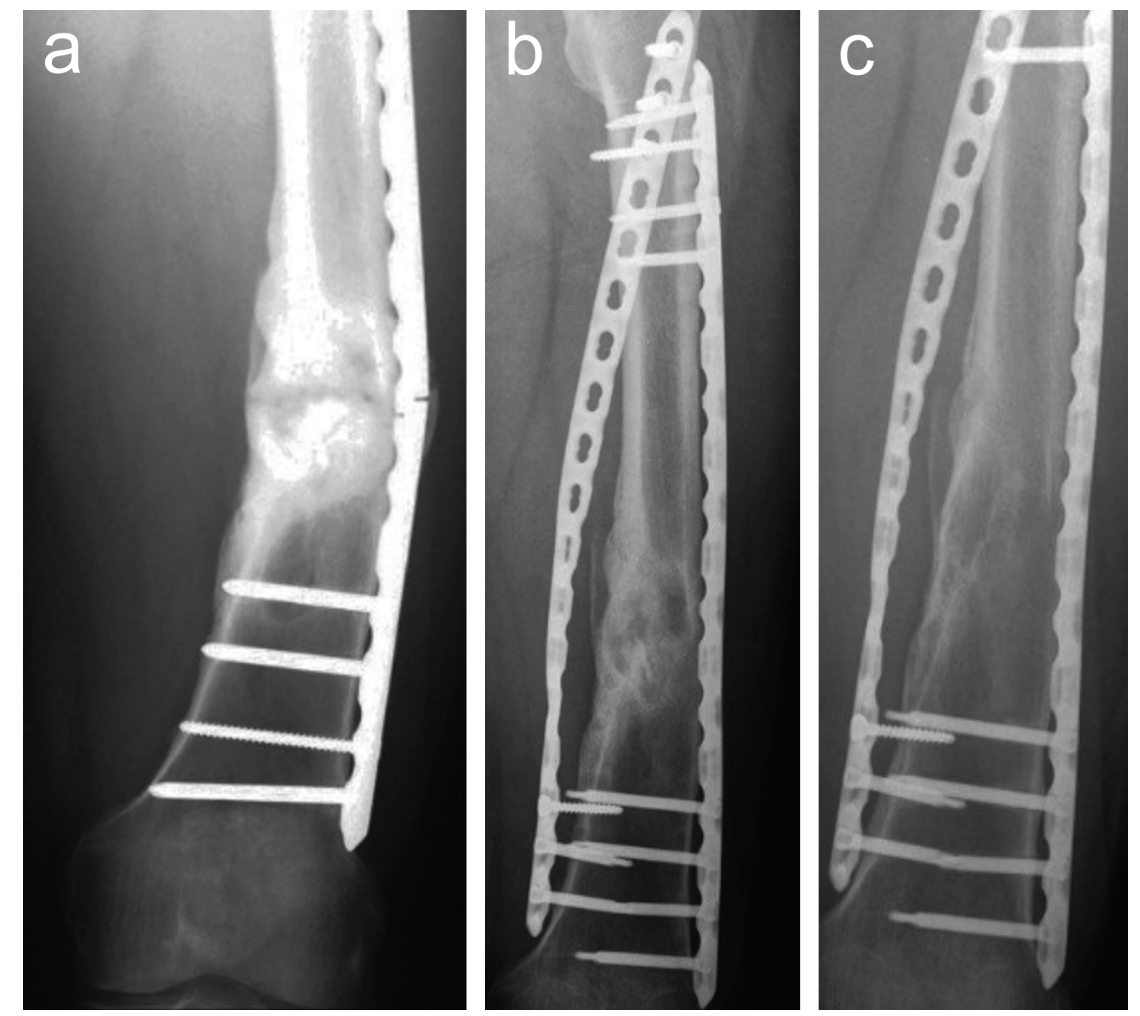

Fig. 1. Radiologic images of a clinical case. (a) Nonunion and lateral locking plate fatigue failure of a comminuted femoral shaft fracture with lacking medial cortical support. (b) Bone healing and callus formation 15 weeks after conversion to lateral locking plate plus helical plate. (c) Continuous bone healing with cortical reformation and absence of plate breakage 145 weeks post-surgery. (C) with permission (source: www.icuc.net). 


\section{Materials and Methods}

Specimens and study groups

Right synthetic femora (\#2230, Synbone, Zizers, Switzerland), made of specially formulated polyurethane foam, comprising of a cancellous inner core with density $170 \mathrm{~kg} / \mathrm{m}^{3}$ and a harder outer shell with density $790 \mathrm{~kg} / \mathrm{m}^{3}$ - simulating cortical bone - were used. The synthetic femora were overly compliant biomechanically as compared to natural human anatomic femora with osteoporotic bone quality (T-score $\leq-2.5$ ). Eight study groups of six specimens each $(n=6)$ were formed as follows (Fig. 2).

- Intact femora without implants (no instrumentation).

- Intact femora instrumented with lateral locking plate.

- Contact transverse shaft osteotomy with lateral locking plate.

- Contact transverse shaft osteotomy with lateral locking plate and helical locking plate.

- Gap transverse shaft osteotomy with lateral locking plate.

- Gap transverse shaft osteotomy with lateral locking plate and helical locking plate.

- Contact transverse shaft osteotomy with long proximal femoral nail.

- Gap shaft osteotomy with long proximal femoral nail.

\section{Instrumentation}

Intact femora

Prior to instrumentation, the intact femora were tested non-destructively as described below, to investigate the stiffness of the non-fractured model and provide baseline values for comparison with the other study groups.

\section{Intact femora with a lateral locking plate}

The centre of the shaft, from the tip of the greater trochanter to the distal end of the lateral condyle, was determined using a caliper rule giving a $215 \mathrm{~mm}$ length on each side. An 18-hole broad curved 4.5/5.0 right titanium Locking Compression Plate (ref. no. 426.682, DePuy Synthes, Zuchwil, Switzerland), precontoured using bending pliers, was mounted to the lateral aspect of the intact femur with the $9_{\text {th }}$ hole - counted from proximal end - over the centre of the shaft. The plate was fixed, using locking screws, in the 4 most proximal and 4 most distal screw holes, resulting in a working length of $190 \mathrm{~mm}$. To reduce the variability in plate attachment, the helical plate was instrumented as described below and removed before testing. Non-destructive testing allowed that all 6 specimens used for plating were instrumented at this point and further used as specimens in the following plating groups. The 6 nailed specimens were instrumented separately, so that in total 12 synthetic bones were used in this study.

\section{Contact osteotomy with a lateral locking plate}

A transverse osteotomy was set in the centre of the femoral shaft and the mechanical testing was repeated. The transverse plane, orthogonal to the shaft axis was determined by a set square. The osteotomy was performed perpendicular to the shaft axis using an oscillating saw with blade thickness of $0.8 \mathrm{~mm}$, producing a gap of $0.8 \mathrm{~mm}$. The fragments made contact under loading.

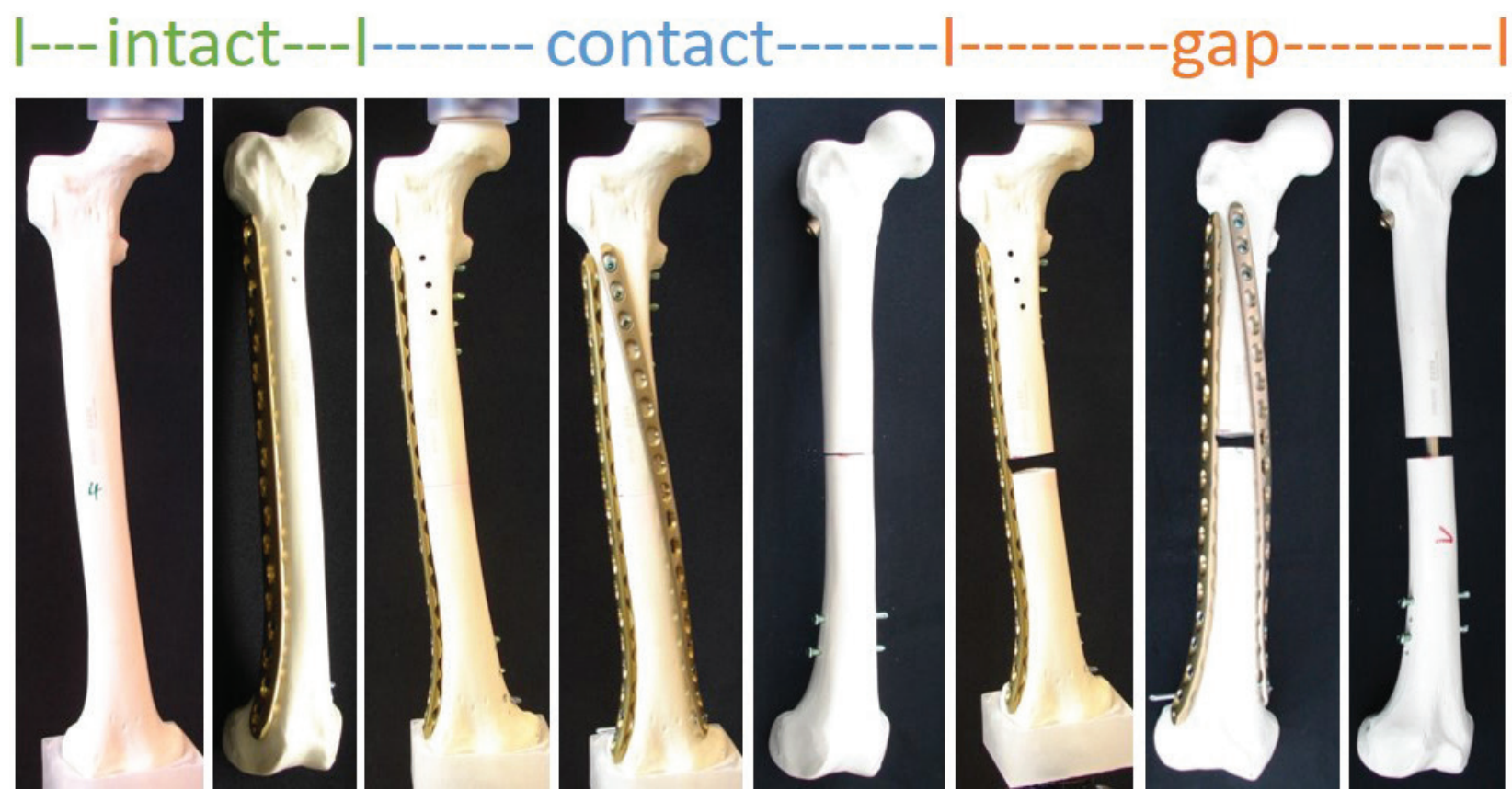

Fig. 2. Photographs of the biomechanically tested constructs. From left to right. (intact) Intact femur without implants and with lateral locking plate. (contact) Contact transverse shaft osteotomy with lateral locking plate; with lateral locking plate and helical locking plate; with long proximal femoral nail. (gap) Gap shaft osteotomy with lateral locking plate; with lateral locking plate and helical locking plate; with long proximal femoral nail. 
Contact osteotomy with lateral locking and helical locking plates

An 18-hole narrow 4.5/5.0 titanium Locking Compression Plate (ref. no. 424.681, DePuy Synthes, Zuchwil, Switzerland), precontoured into a helical shape using bending irons and pliers, was fixed to the anterior aspect of the femur proximally and to its anteromedial aspect distally with 3 locking screws, each at both plate ends, providing a working length of $230 \mathrm{~mm}$ (helical plate).

Gap osteotomy with a lateral locking plate

After mechanical testing, the helical plate was removed, and a $10 \mathrm{~mm}$ transverse gap was created by setting a second osteotomy in parallel and $10 \mathrm{~mm}$ proximally to the first one. The femur was mechanically tested with the lateral locking plate left in place. The fragments did not make contact under loading.

Gap osteotomy with lateral locking and helical locking plates

The helical plate was remounted, and mechanical testing was repeated.

\section{Contact osteotomy with a nail}

Another 6 femora were instrumented with a right $130^{\circ}$ long Proximal Femoral Nail Antirotation (PFNA) of $\varnothing 10 \mathrm{~mm}$ and $360 \mathrm{~mm}$ length (ref. no. 04.027.226, DePuy Synthes, Zuchwil, Switzerland) including a 95 mm PFNA-II blade (ref. no. 04.027.014, DePuy Synthes, Zuchwil, Switzerland) according to the manufacturer's guidelines. Before nail insertion, the medullary canal was reamed up to $12 \mathrm{~mm}$ diameter and a transverse osteotomy was set in the centre of the femoral shaft as described above. Distal locking was performed with two self-tapping $4.9 \mathrm{~mm}$ locking bolts (ref. no. 459.500, DePuy Synthes, Zuchwil, Switzerland).

\section{Gap osteotomy with a nail}

A $10 \mathrm{~mm}$ wide gap was produced, located as described above.

\section{Mechanical testing (stiffness)}

Mechanical testing was performed using a servohydraulic mechanical test system (Bionix 858; MTS Systems, Eden Prairie, MN, USA) equipped with a $25 \mathrm{kN} / 250 \mathrm{Nm}$ load cell at an accuracy of $0.38 \%$ at $250 \mathrm{~N}$ force and an accuracy of $0.52 \%$ at $3 \mathrm{Nm}$ torque, representing an accuracy of approximately $1 \mathrm{~N} / 0.02 \mathrm{Nm}$. For axial loading, the femoral head of the specimen was attached to the actuator of the test frame proximally using a custom-made polymethylmethacrylate (PMMA, SCSBeracryl D-28, Suter Kunststoffe AG, Fraubrunnen, Switzerland) mould, forming a ball-and-socket joint with unconstrained degrees of freedom. The femoral shaft was positioned at $8^{\circ}$ valgus relative to the load axis of the machine, ensuring a physiological load bearing (Bergmann et al., 2001) of the construct with axial compression force as the main load (axial bending) (Fig. 3a). For torsional loading, the proximal part of the femur was encased in a custom-made PMMA mould connected by a cardan joint to the machine actuator with the femoral shaft axis oriented along the torsional axis (Fig. 3b). For both axial and torsional loading, the distal part of the femur was encased in a custom-made PMMA mould, connected by a cardan joint to the load cell and the test frame.

Axial bending loading was performed using a quasi-static force ramp from $20 \mathrm{~N}$ to $100 \mathrm{~N}$ at a rate of $10 \mathrm{~N} / \mathrm{s}$. Torsional loading was performed in internal rotation using a quasi-static torque ramp from $0 \mathrm{Nm}$ to $2 \mathrm{Nm}$ at a rate of $0.2 \mathrm{Nm} / \mathrm{s}$. Preliminary tests revealed that the applied forces and moments were within the linear elastic range of the constructs. Due to overly compliant bone, the applied forces
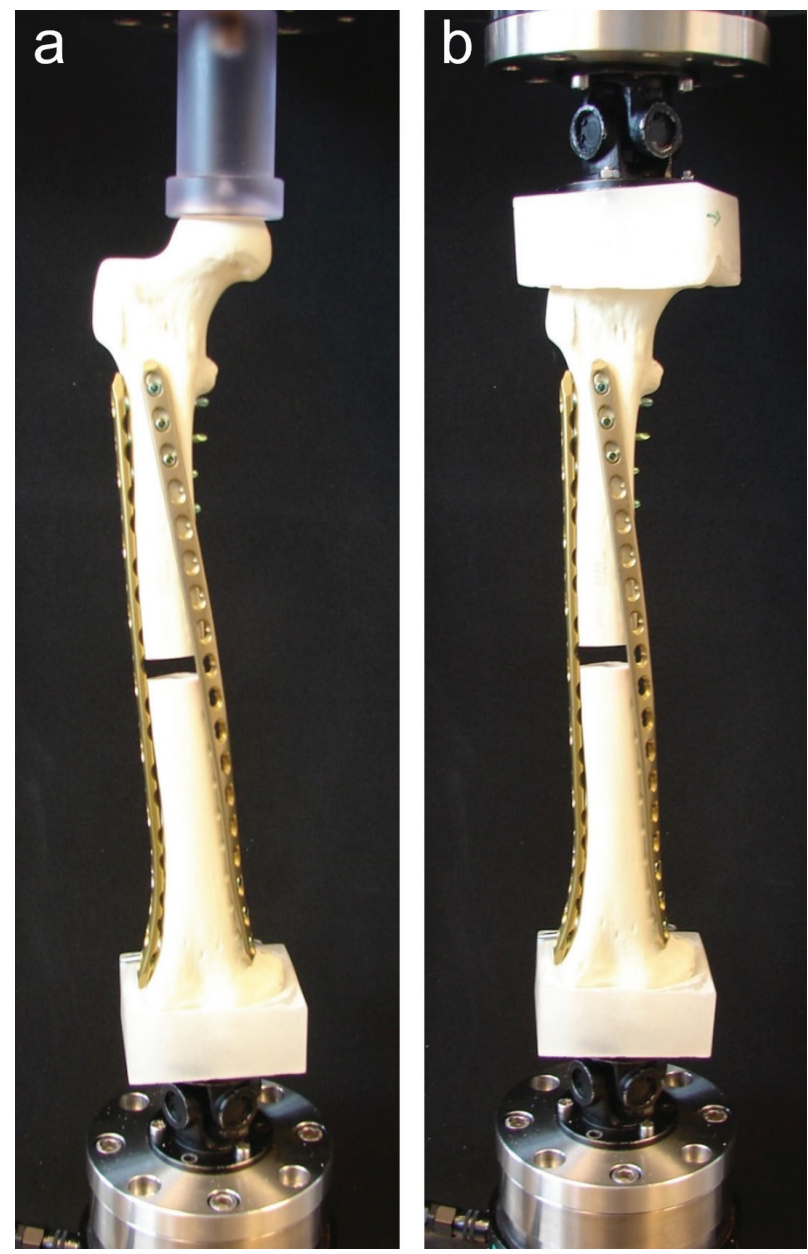

Fig. 3. Setups with specimens mounted for biomechanical testing. (a) For axial loading, the femoral shaft was positioned in $8^{\circ}$ valgus relative to the load axis of the machine. The femoral head was a part of a ball-and-socket joint, using a spherical mould connecting the specimen to the machine actuator. (b) For torsional loading, the femoral shaft axis was oriented along the torsional axis of the machine and the proximal end of the bone was connected to the actuator by PMMA embedding and a cardan joint. Distally, for both setups, the specimen was connected to the load cell using a PMMA mould and a cardan joint. 
and moments were considered reasonable. The axial and torsional ramps were run consecutively for three cycles. The third cycle was used for stiffness evaluation to allow settling of the construct during the first two cycles.

\section{Mechanical testing (strain assessment)}

For surface strain assessment of the implants, an axial load of $200 \mathrm{~N}$ was applied using the same test setup (Fig. 3a). Strain was recorded at the lateral plate, with and without application of a helical plate, and on the helical plate itself, respectively. Three strain measurements were performed separately under an identical load.

The major principal strain was measured on the surface of the plate using a contactless full-field deformation technology (Aramis SRX, GOM GmbH, Braunschweig, Germany). The implant surface was sprayed to create a fine black speckle pattern on a white background. The strain level was evaluated using the stereographic optical measurement principle with digital image correlation. Both spatial and temporal filters were used to average the strain values. For the temporal filter, 4 images before and after the current image were used and the corresponding strains of each node were averaged. With an image acquisition rate of $50 \mathrm{~Hz}$ (0.02 s/image), the 9 evaluated images corresponded to a time window of $0.18 \mathrm{~s}$, which was negligible to the quasi-static loading rate. The spatial filter was used to average the values in the neighbourhood of the 4 th order, meaning that values in the neighbourhood from the current measuring point to the 4 next surrounding measuring points were averaged. Strain accuracy of the measurement system was up to $0.05 \%$ as measured on a static reference object in an undeformed state.

\section{Data acquisition and analysis}

An effect size of 1.80 was calculated for $n=6$ per group at a power of 0.8 using $\mathrm{G}^{*}$ Power software package (Release 3.1.9.6, Heinrich-Heine University, Düsseldorf, Germany) to show test sensitivity (Faul et al., 2007). Machine data in terms of axial force, torque, axial, and torsional displacements were recorded from the test system's transducers at a sampling rate of $128 \mathrm{~Hz}$. A custom-made software (Matlab R2020b; MathWorks, Natick, MA, USA) was used for data evaluation. Construct stiffness was determined from the linear part of the 3 rd quasi-static ramp in the interval from $50 \mathrm{~N}$ to $80 \mathrm{~N}$ for axial bending loading and from $0.5 \mathrm{Nm}$ to $1.5 \mathrm{Nm}$ for torsional loading. Statistical analysis was performed using an SPSS software package (IBM SPSS Statistics, V27, IBM, Armonk, NY, USA). Normal distribution within each study group was screened and proven using ShapiroWilk Test. Significance of differences between the study groups in terms of axial bending stiffness and torsional stiffness were identified using One-Way Analysis of Variance (ANOVA). Bonferroni post hoc correction was applied for multiple comparisons. Significance level was set at 0.05 for all statistical tests.

\section{Results}

Using compliant synthetic bone, axial stiffness increased significantly in comparison with the intact bone when a plate was attached to it (104\% increase, $p<0.01)$ and when the contact groups were stabilised with a plate $(46 \%$ increase, $p<0.01)$ or a nail (204 \% increase, $p<0.01)$ (Fig. 4). When the gap was bridged by a single lateral plate, axial stiffness was significantly lower compared with the intact synthetic bone ( $49 \%$ decrease, $p<0.01$ ) as well as with all other groups (Table 1). By adding a helical plate to the lateral plate in both contact and gap situations, axial stiffness increased significantly compared with the corresponding single lateral plate fixation (86\% increase in the contact groups, $436 \%$ in the gap groups, both $p<0.01)$. The addition of a helical plate to the lateral plate increased torsional stiffness significantly compared with a single lateral plate in both the contact and gap groups (29\% increase for both, $p<0.01$ ) (Fig. 5). Torsional stiffness of the construct with both a lateral and helical plate in the gap situation was significantly higher compared to

Table 1. Axial and torsional stiffness in terms of mean value and standard deviation for intact, contact and gap study groups $(n=6)$.

\begin{tabular}{|c|c|c|}
\hline \multirow{2}{*}{ Study groups } & \multicolumn{2}{|c|}{ Stiffness } \\
\cline { 2 - 3 } & Axial [N/mm] & Torsional [Nm/ $\left.{ }^{\circ}\right]$ \\
\hline Intact \\
\hline No implant & $71 \pm 2$ & $0.7 \pm 0.1$ \\
\hline Lateral plate & $145 \pm 6$ & $1.1 \pm 0.1$ \\
\hline \multicolumn{3}{|c|}{ Contact } \\
\hline Lateral plate & $104 \pm 7$ & $0.7 \pm<0.1$ \\
\hline Lateral and helical plates & $193 \pm 14$ & $0.9 \pm 0.1$ \\
\hline Nail & $216 \pm 17$ & $0.8 \pm 0.1$ \\
\hline \multicolumn{3}{|c|}{ Gap } \\
\hline Lateral plate & $36 \pm 1$ & $0.7 \pm<0.1$ \\
\hline Lateral and helical plates & $193 \pm 17$ & $0.9 \pm 0.1$ \\
\hline Nail & $192 \pm 14$ & $0.7 \pm<0.1$ \\
\hline
\end{tabular}




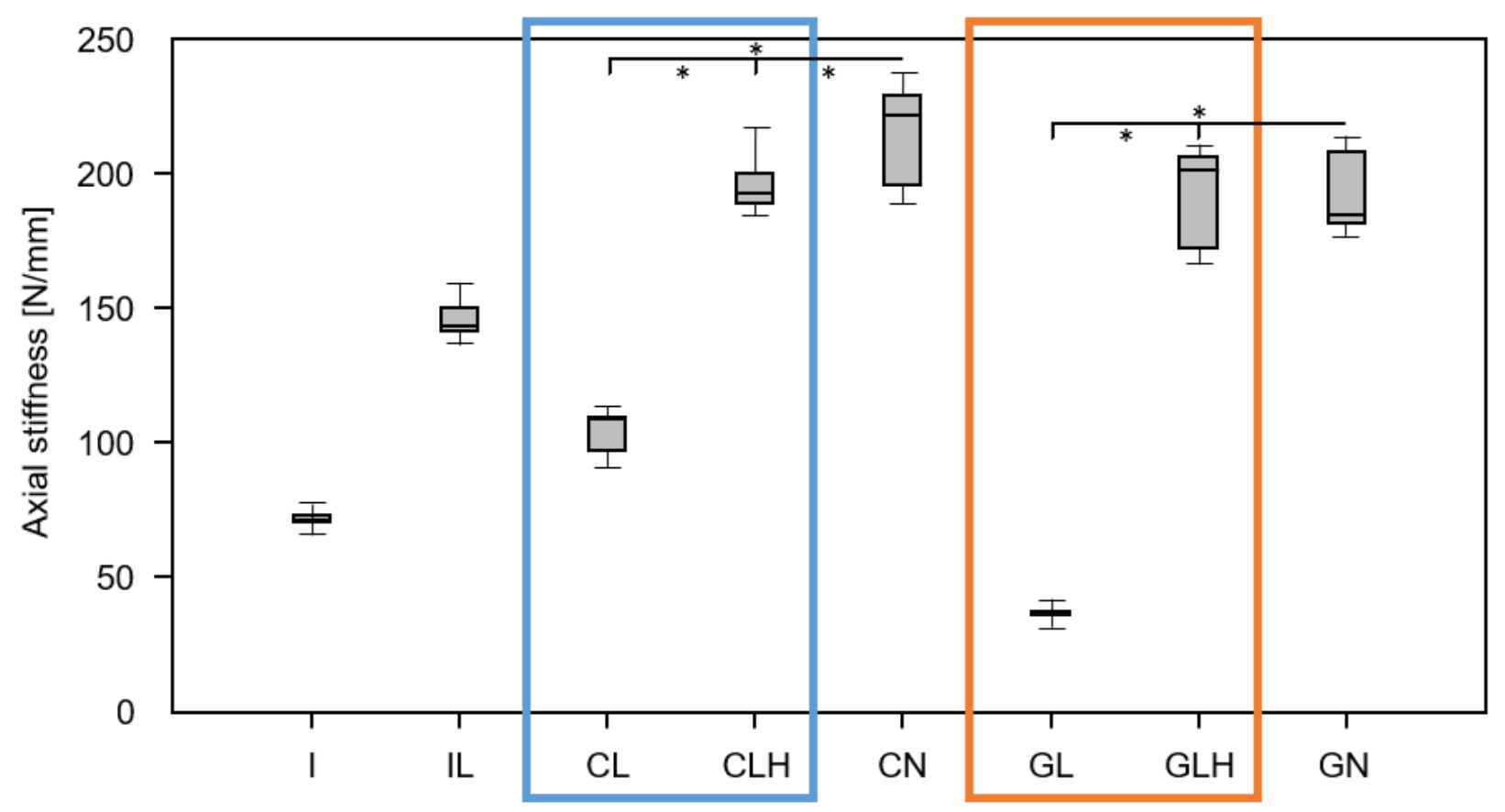

Fig. 4. Boxplots presenting axial stiffness of the tested groups. I: intact, IL: intact with lateral plate, CL: contact with lateral plate, CLH: contact with lateral plate and helical plate, CN: contact with nail, GL: gap with lateral plate, GLH: gap with lateral plate and helical plate, GN: gap with nail. Significant differences $(p<0.05)$ of selected comparisons are marked with asterisks $\left(^{*}\right)$. Due to the lower lateral plate's inherent stiffness, the effect of adding a helical plate is stronger when a gap is bridged (orange frame, right). In a contact construct under axial loading (blue frame, left), the fracture fragments add to the inherent stiffness of a lateral plate, while the effect of adding a helical plate is less pronounced.

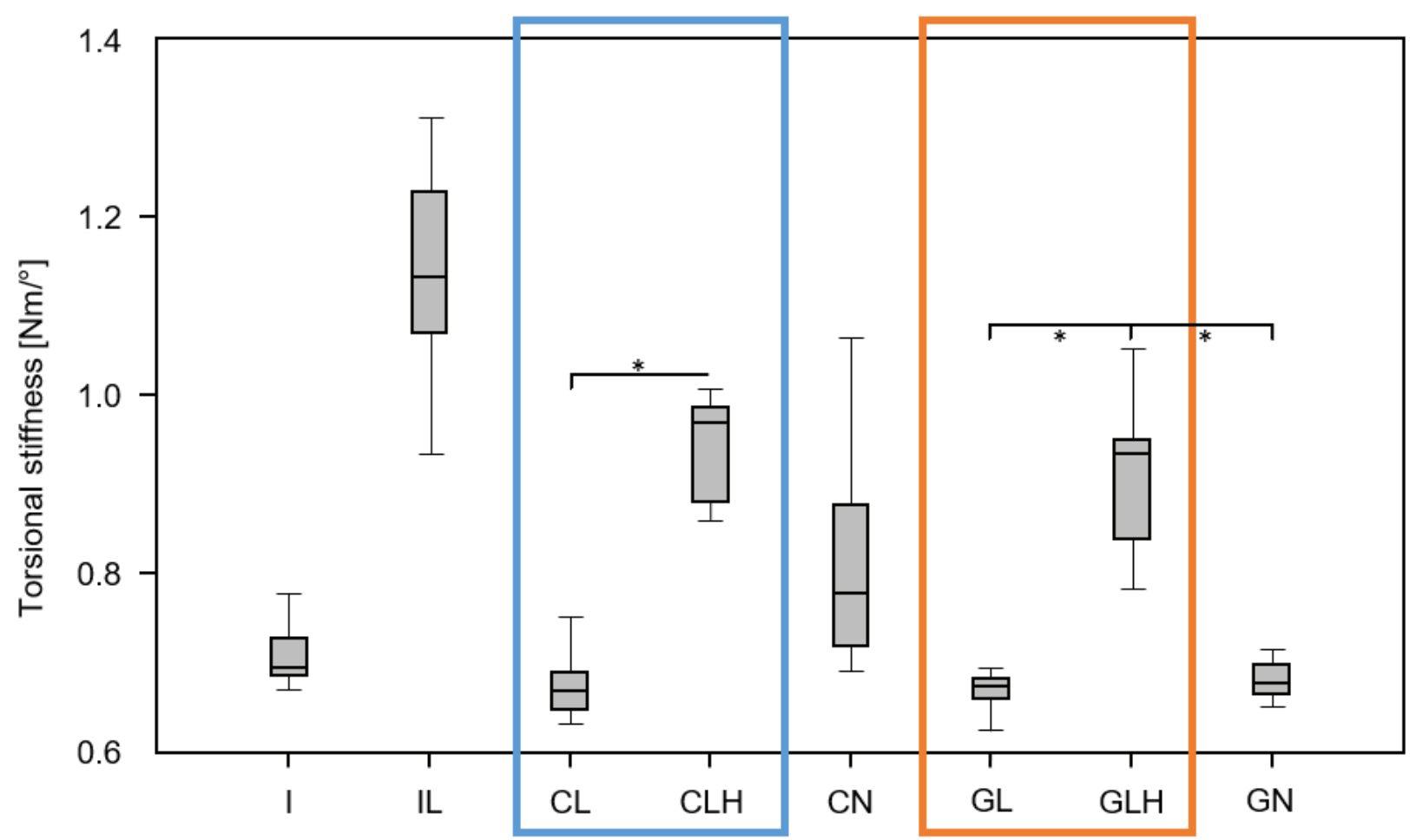

Fig. 5. Boxplots presenting torsional stiffness of the tested groups. I: intact, IL: intact with lateral plate, CL: contact with lateral plate, CLH: contact with lateral plate and helical plate, CN: contact with nail, GL: gap with lateral plate, GLH: gap with lateral plate and helical plate, GN: gap with nail. Significant differences $(p<0.05)$ of selected comparisons are marked with asterisks $\left({ }^{*}\right)$. Transverse fractures do not provide abutment for torsional moment with respect to the femoral shaft. The effect of the helical plate is independent of contact (blue frame, left) or gap (orange frame, right) construct. 
the gap situation stabilised by a nail (29\% increase, $p<0.01)$. Overall, constructs with both a lateral and a helical plate delivered similar axial and torsional stiffness independently of the contact or gap situations. Representative curves for axial and torsional loading of the specimens in all groups are given in Fig. 7 and Fig. 8, respectively.

Maximum surface strain of the single lateral locking plate with gap under axial load of $200 \mathrm{~N}$ reached values of up to $0.3 \%$ adjacent to the plate holes located at the gap (Fig. 6). Following additional anteromedial helical plating with a gap, the surface strain of both the lateral and helical plates exhibited values of less than $0.1 \%$, being less than $1 / 3$ of the maximum surface strain observed with the single lateral plate configuration.

\section{Discussion}

Compared with single locked plating, the addition of a helical plate significantly increased axial (86\% contact, $436 \%$ gap) and torsional (29\% contact, $29 \%$ gap) construct stiffness. Helical plating of contact and gap constructs achieved similar stiffness values. Fracture configuration plays a minor role for axial and torsional construct stiffness if a helical plate is applied. Due to missing cortical support at the medial fracture site, the effect of adding a helical plate under axial load is more pronounced when a gap is bridged. Especially in comminuted fractures and fractures with an open fracture gap, bone healing depends on the mechanical conditions within the gap, determined by the stability of the osteosynthesis. As demonstrated by the results, an intramedullary load carrier such as the nail provides axial stiffness comparable to a helical-plate construct. This study was intended to provide an insight into the effect of helical plating, using a simple biomechanical model. Although many shaft fractures can be stabilised by intramedullary nailing, this osteotomy model was considered to be realistic for mimicking the effect of a gap situation in comminuted fractures on plate loading. A diaphyseal model was used according to its original description (Fernandez Dell'Oca, 2002a). The helical-plate technique might also be applied for fractures of the distal $1 / 3$ of the femur, where many defect fractures occur and often need a second plate for sufficient fixation. Due to the simplicity of the model, the results might be transferable to a distal fracture location. Since the anatomical axis of the femur is inclined at $8-10^{\circ}$ laterally to the load axis of the leg, a medial bending moment is created in the femur under axial loading (Link and Babst, 2012), which has to be counterbalanced by the implant. In a construct with a single lateral locking plate and
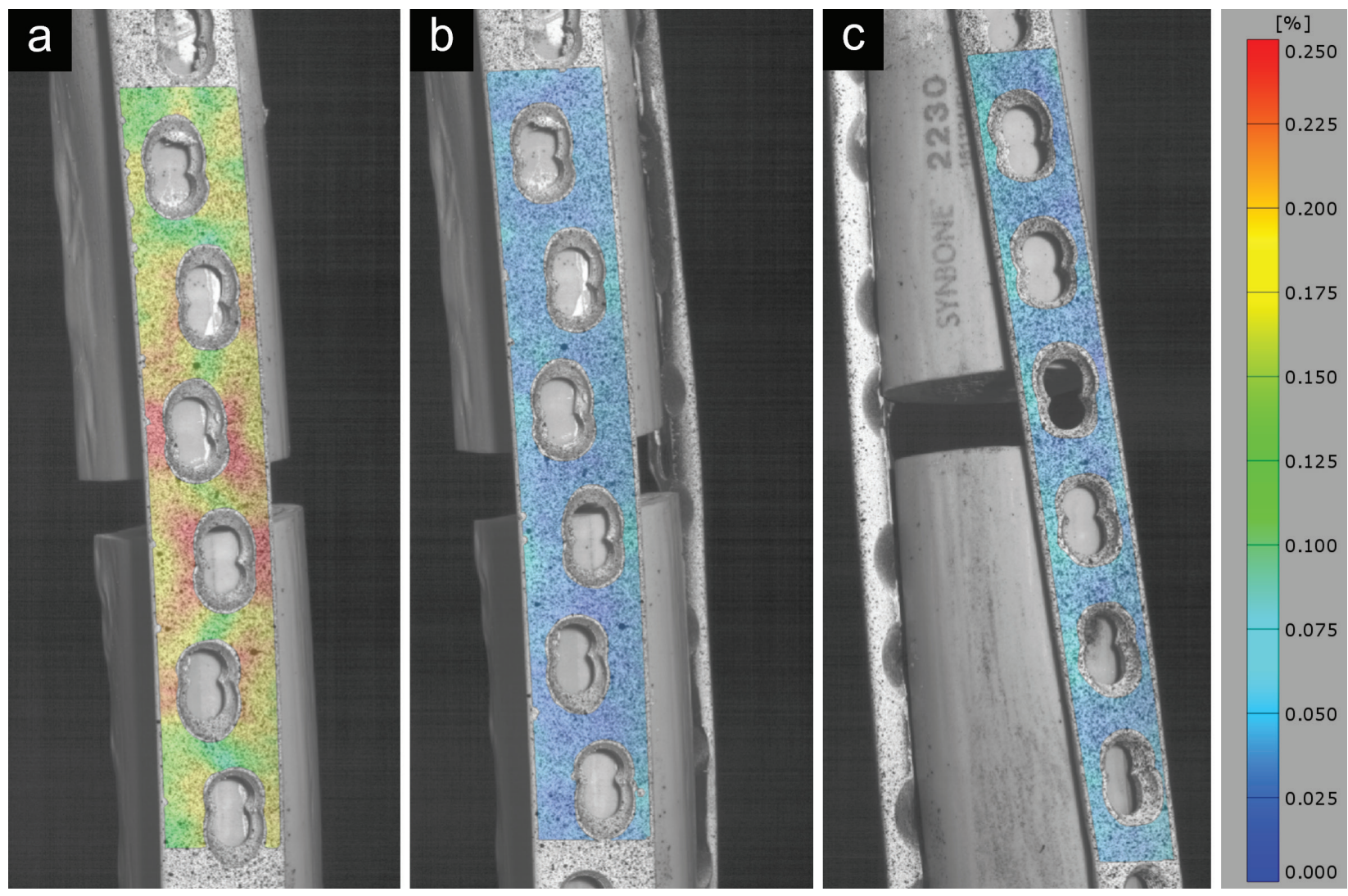

Fig. 6. Maximum major principal strain on the surface of a single lateral locking plate spanning a gap and loaded at $200 \mathrm{~N}$. (a) The red colour indicates a maximum surface strain above $0.3 \%$ with a single lateral locking plate. (b) Adding an anteromedial helical plate to the lateral locking plate decreases the surface strain on the lateral plate to a level below $0.1 \%$ (blue colour). (c) Surface strain of the anteromedial helical plate itself ranges below $0.1 \%$ (blue colour). 
bone contact, reduced tight-fitting fracture fragments provide cortical abutment and increase the stiffness of the construct. The stress in the lateral plate is up to 50-fold lower in a stable fracture with a closed fracture gap under compression, compared with an open fracture gap without cortical support (Moazen et al., 2013). Depending on the fracture configuration, axial forces and torsional moments could be transmitted by fracture fragment interdigitation. Strain visualisation shows that by adding a helical plate, both the lateral and helical plates are similarly strained. However, the surface strain on the plate is dependent on the implantation quality, plate type, plate, and screw positions. An ideal construct

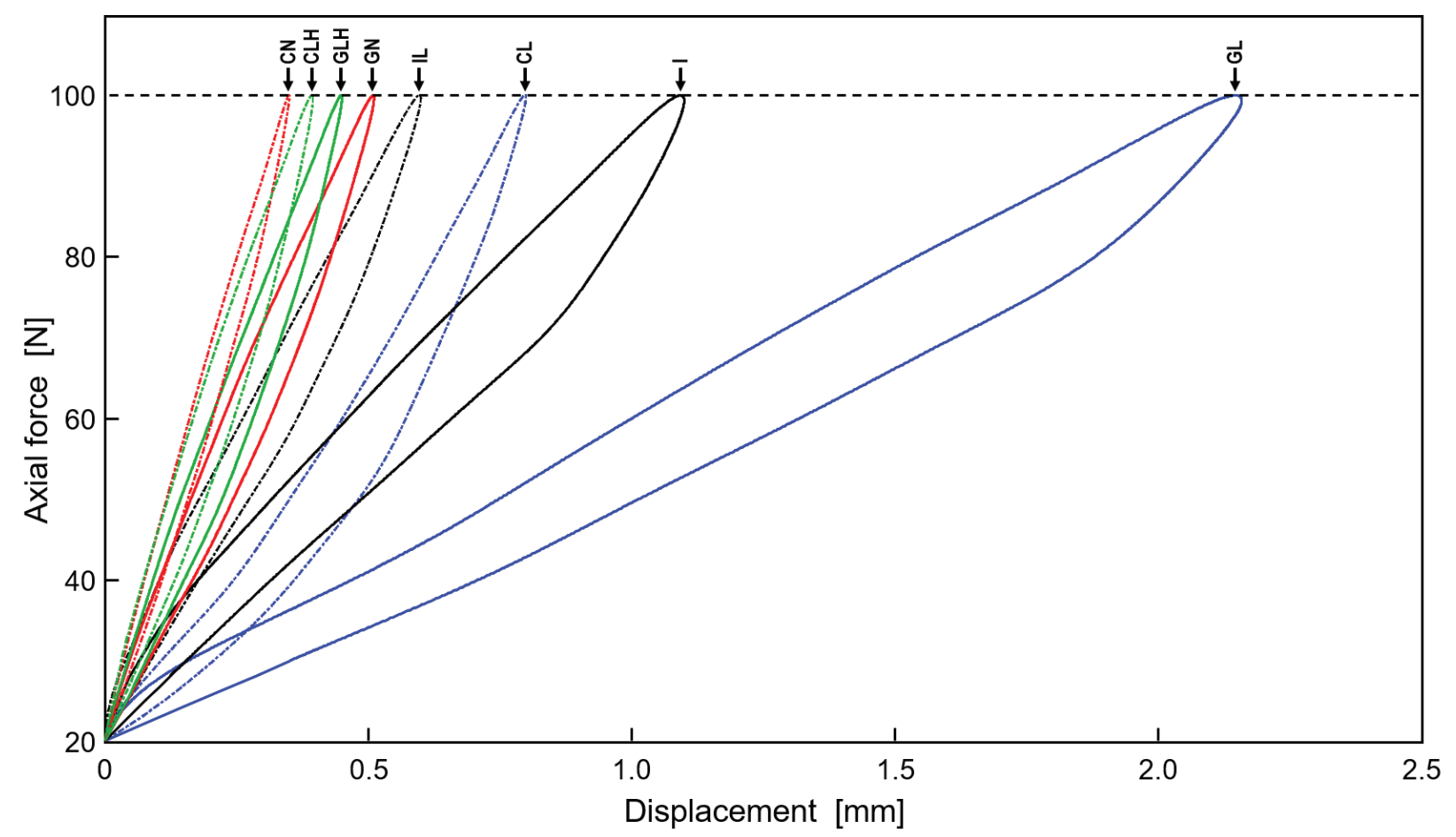

Fig. 7. Representative curves for axial loading of the specimens in all groups. I: intact, IL: intact with lateral plate, CL: contact with lateral plate, CLH: contact with lateral plate and helical plate, CN: contact with nail, GL: gap with lateral plate, GLH: gap with lateral plate and helical plate, GN: gap with nail.

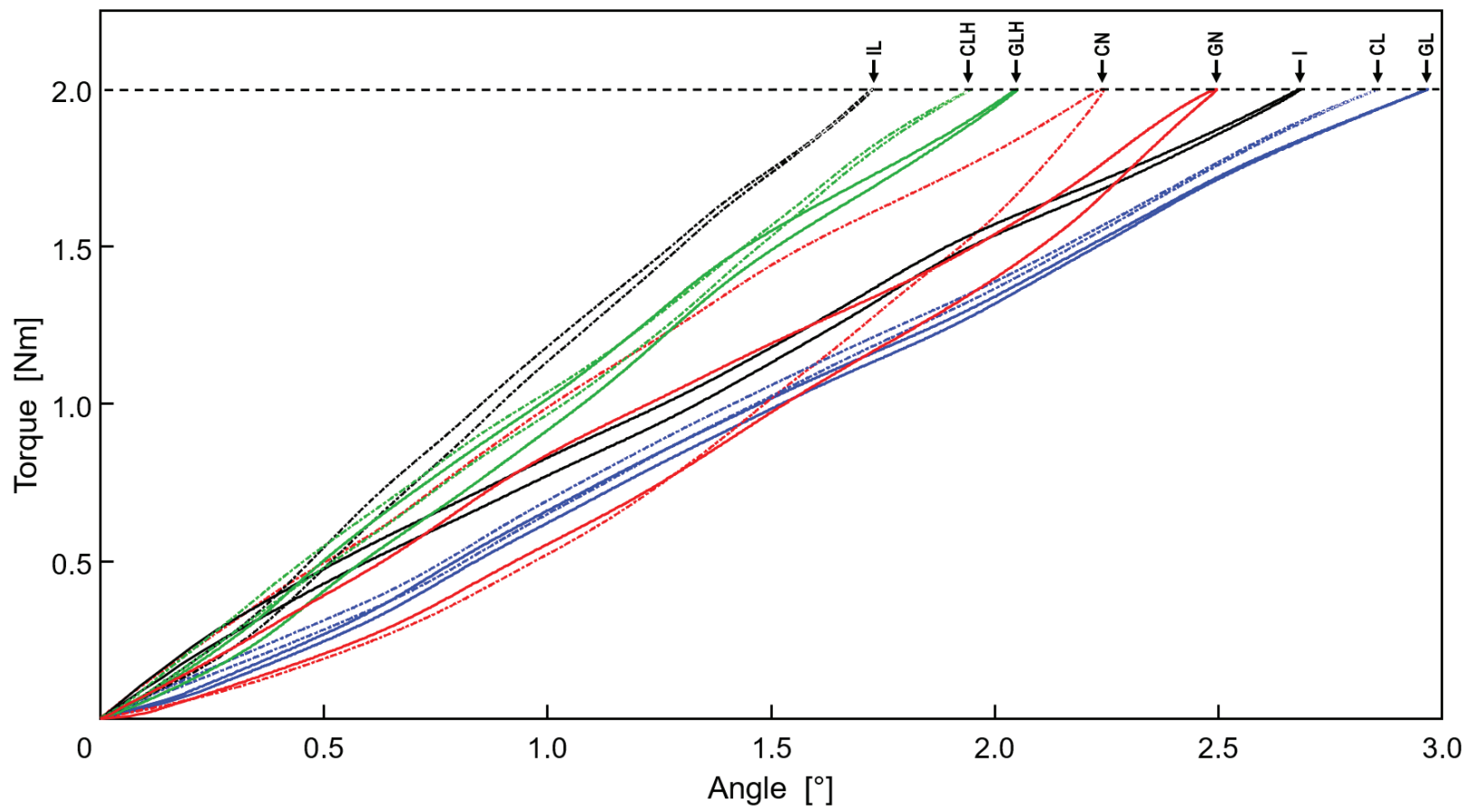

Fig. 8. Representative curves for torsional loading of the specimens in all groups. I: intact, IL: intact with lateral plate, CL: contact with lateral plate, CLH: contact with lateral plate and helical plate, CN: contact with nail, GL: gap with lateral plate, GLH: gap with lateral plate and helical plate, GN: gap with nail. 
would position the second plate on the medial cortex, which is not feasible for the proximal femur region due to the surgical approach needed. Shared load in double plating reduces the stress in the lateral plate, compared to single lateral plating.

It is important to differentiate between the following 2 mechanical conditions in lateral locking plate osteosynthesis of the femur.

If the fracture gap is closed or closes under load, the lateral plate acts as a tension band (Sommer et al., 2003). Longer plates with longer working length should be applied to minimise pull-out forces of the screws and to minimise fatigue failure of the plate. The plate section at the fracture site is more deformed and a greater bending moment in the plate is necessary to achieve medial cortical contact if the screws are located close to the fracture and the working length is short (Sommer et al., 2003). The lateral plate should be placed directly in contact with the shaft so that the plate buttresses the bone during bending.

If the fracture gap does not close medially under load, the full bending moment created under load acts on the plate without load sharing with the bone, so that repeated plate bending can induce its breakage. If a medial cortical abutment is missing, a longer working length of the plate will not reduce the bending moment acting upon it and will not protect the plate from breakage. This phenomenon is even more severe if the plate is heavily bent in a fracture gap situation, so that the bony cortex at the fracture site levers on the plate. The bony contact acts as a stress riser and the working length is now determined by the width of the fracture gap, resulting in an accelerated failure event (MacLeod and Pankaj, 2018). A longer working length only renders the construct weaker in this situation. In the case of a gap situation or comminuted fracture, the strain on the plate can be reduced by applying a shorter working length. A long working length should be used in situations where the fracture gap closes under load while a shorter working length should be applied in situations where the fracture gap does not close during loading. With the addition of a medial helical plate the loading conditions change, since in case of ideal helical plate placement strictly on the bone, the bending moment of the lateral plate is completely neutralised. Similarly, a reduction of surface strains was observed in the lateral plate to less than $1 / 3$ of the initial values by adding an anteromedial helical locking plate. Adding a helical plate to the lateral plate construct provided similar deformation of the 2 plates, indicated by the same level of their maximal surface strains. In agreement with the surface strain evaluation, a finite element analysis in a gap periprosthetic fracture model shows a stress reduction of $1 / 3$ up to $1 / 4$ of the initial stress within the lateral plate, if double plating is used instead of single lateral locked plating (Moazen et al., 2014).

Transverse fractures, such as used in the presented fracture model, do not provide bony load transfer for torsional moments in both the contact and gap situations. Torsion around the femoral shaft axis, being a typical moment appearing during stair climbing, was applied in the testing setup. This has to be considered critical for fracture healing in single lateral locked plating since shear forces exist in the fracture plane (Todorov et al., 2018). Besides torsion, shear movement at the fracture gap arises at plate bending under load (Elkins et al., 2016). Analysis of a cohort of 66 distal femoral fractures fixed with lateral locking plates at different working lengths, together with a finite element simulation, revealed that longitudinal movement correlated positively with callus formation - whereas shear movement correlated negatively with it (Elkins et al., 2016). Working lengths of more than $80 \mathrm{~mm}$ demonstrate an exponential increase in shear movement relative to the longitudinal movement at the fracture gap (Elkins et al., 2016). Titanium plates provide a higher compliance and thus exhibit larger degrees of bending compared to stainless steel plates, so that a titanium locking plate with a working length below $80 \mathrm{~mm}$ is recommended for optimal fixation (Elkins et al., 2016). To point out the function and the effect of the helical plate upon construct stiffness and plate surface strain, a longer working length of $190 \mathrm{~mm}$ was used for the lateral plate, which would not be considered in a clinical situation.

Various studies have demonstrated the clinical effectiveness of additional medial plating (Henderson et al., 2011; Holzman et al., 2016; Khalil Ael and Ayoub, 2012; Sanders et al., 1991; Steinberg et al., 2017; Ziran et al., 2002). In a case series of 21 distal femoral nonunions initially treated with lateral plating, the addition of a medial locking plate and autogenous bone grafting led to radiological osseous union in 20 fractures (Holzman et al., 2016). Bone grafting must be included in nonunion treatment or as secondary procedure in double plating if primary callus formation is impeded (Holzman et al., 2016). Anatomical studies reveal a low risk for medial plate application if vascular anatomy is known and respected (Jiamton and Apivatthakakul, 2015; Kim et al., 2014). The safe zone for medial plate positioning starts $80 \mathrm{~mm}$ below the lesser trochanter (Kim et al., 2014) or distal to $60 \%$ of the femoral length (Jiamton and Apivatthakakul, 2015). For this reason and to avoid placement onto the origin of the rectus muscle, a helical shaped form has been developed to allow submuscular minimally-invasive plate positioning from proximal anterolateral to distal medial (Fernandez Dell'Oca, 2002a).

The current study had several limitations. It focused on the comparison of different plating techniques to investigate the mechanical implant behaviour depending on the fracture gap situation and not to explore the implant anchorage to bone. To exclude the influence of varying bone quality, the study was limited to a synthetic bone model, which allows comparison in-between different constructs; however, the used intact bone was overly compliant. 
To compensate for the disadvantage of increased deformation of the synthetic bone specimen itself, a group of intact bone with applied lateral locking plate was included to provide a further reference for inter-group comparison. Drill holes and repetitive testing might have compromised mechanical stability of the bone. Since testing was performed below the level of plastic deformation of the synthetic bone, this effect was considered to be negligible. The effect of the helical plate was examined at a very low axial load (100 N) and torsional moment (2 Nm) levels, being below the clinical levels under full weight bearing. Because of the overly-compliant synthetic bone specimens used, they had to be adapted. Due to the gap, construct stiffness was mainly influenced by the plate stiffness, which was the goal of the investigation. In clinical scenarios, plate breakage and not screw cut-out is the most observed failure of lateral locking plate fixations in femoral shaft defect fractures. Especially in the gap groups, increased stiffness of the helical plate construct was significant compared to lateral plating and these findings were considered relevant despite the low load and moment levels. Selecting a long working length of the lateral plate, the small gap in the lateral plate contact group was closed under preload reaching a linear response curve. Differences in stiffness between the groups were determined in the linear range of the load curve which allowed determination and comparison of construct stiffness. A very long working length of $190 \mathrm{~mm}$ was chosen for the lateral plate to create a less stiff construct, mimicking plate fixations of defect fractures. The effect of the helical plate was more pronounced in this configuration. Although not applied clinically in non-gap situations, this working length was kept for the non-gap (contact) groups to provide inter-group comparability.

The axial and torsional construct stiffness delivered indirect information about the expected movement at the fracture site, which is an important parameter for bone healing. However, detailed differentiation between longitudinal and shear movement was not performed. Fatigue behaviour and failure mode have not been investigated and would have required further cyclic testing.

As a further limitation, the surface strain images of the plates were spatially filtered to remove edge artefacts. The surface strains of the double-plated configuration were small and thus may have been affected by the accuracy of the system. However, the relative difference of the strains between the singleand double-plated configurations could be assessed, which was the aim of this analysis.

Helical plates are applied in unstable fractures for strain reduction in the lateral plate and reduction of interfragmentary movements at the fracture site. The addition of a helical plate drastically improves the mechanical situation, providing a higher loadbearing capacity and reduces the risk of hardware failure and nonunion.

\section{Conclusions}

The helical plate, added to a single lateral plate, increases the overall stiffness independent of the gap situation. It should be considered in very demanding situations when treating gap or defect fractures, where single plate osteosynthesis provides inadequate stiffness for fracture healing and induces nonunion. Furthermore, the helical plate reduces surface strains of the single plate, protecting the latter from failure.

\section{Acknowledgements}

This investigation was performed at the AO Research Institute Davos, Switzerland, with the assistance of the AO Foundation via the AO Technical Commission. The authors are not compensated and there are no other institutional subsidies, corporate affiliations, or funding sources supporting this work unless clearly documented and disclosed.

\section{References}

Beeres FJP, Emmink BL, Lanter K, Link BC, Babst R (2020) Minimally invasive double-plating osteosynthesis of the distal femur. Oper Orthop Traumatol 32: 545-558.

Bergmann G, Deuretzbacher G, Heller M, Graichen F, Rohlmann A, Strauss J, Duda GN (2001) Hip contact forces and gait patterns from routine activities. J Biomech 34: 859-871.

Bottlang M, Schemitsch CE, Nauth A, Routt M, Jr., Egol KA, Cook GE, Schemitsch EH (2015) Biomechanical concepts for fracture fixation. J Orthop Trauma 29 Suppl 12: S28-S33.

Comiskey DP, Macdonald BJ, McCartney WT, Synnott K, O'Byrne J (2010) The role of interfragmentary strain on the rate of bone healing - a new interpretation and mathematical model. J Biomech 43: 2830-2834.

Ebraheim NA, Liu J, Hashmi SZ, Sochacki KR, Moral MZ, Hirschfeld AG (2012) High complication rate in locking plate fixation of lower periprosthetic distal femur fractures in patients with total knee arthroplasties. J Arthroplasty 27: 809-813.

Elkins J, Marsh JL, Lujan T, Peindl R, Kellam J, Anderson DD, Lack W (2016) Motion predicts clinical callus formation: construct-specific finite element analysis of supracondylar femoral fractures. J Bone Joint Surg Am 98: 276-284.

Faul F, Erdfelder E, Lang AG, Buchner A (2007) $\mathrm{G}^{*}$ Power 3: a flexible statistical power analysis program for the social, behavioral, and biomedical sciences. Behav Res Methods 39: 175-191.

Fernandez Dell'Oca AA (2002a) The principle of helical implants. Unusual ideas worth considering. Injury 33 Suppl 1: SA1-SA27. 
Fernandez Dell'Oca AA (2002b) The principle of helical implants: unusual ideas worth considering. Case studies. Injury 33 Suppl 1: SA29-SA40.

Henderson CE, Kuhl LL, Fitzpatrick DC, Marsh JL (2011) Locking plates for distal femur fractures: is there a problem with fracture healing? J Orthop Trauma 25 Suppl 1: S8-S14.

Hoffmann MF, Jones CB, Sietsema DL, Koenig SJ, Tornetta P, 3rd (2012) Outcome of periprosthetic distal femoral fractures following knee arthroplasty. Injury 43: 1084-1089.

Holzman MA, Hanus BD, Munz JW, O'Connor DP, Brinker MR (2016) Addition of a medial locking plate to an in situ lateral locking plate results in healing of distal femoral nonunions. Clin Orthop Relat Res 474: 1498-1505.

Jiamton C, Apivatthakakul T (2015) The safety and feasibility of minimally invasive plate osteosynthesis (MIPO) on the medial side of the femur: a cadaveric injection study. Injury 46: 2170-2176.

Khalil Ael S, Ayoub MA (2012) Highly unstable complex C3-type distal femur fracture: can double plating via a modified Olerud extensile approach be a standby solution? J Orthop Traumatol 13: 179-188.

Kim JJ, Oh HK, Bae JY, Kim JW (2014) Radiological assessment of the safe zone for medial minimally invasive plate osteosynthesis in the distal femur with computed tomography angiography. Injury 45: 19641969.

Link BC, Babst R (2012) Current concepts in fractures of the distal femur. Acta Chir Orthop Traumatol Cech 79: 11-20.

Liporace FA, Yoon RS (2019) Nail plate combination technique for native and periprosthetic distal femur fractures. J Orthop Trauma 33: e64-e68.

MacLeod AR, Pankaj P (2018) Pre-operative planning for fracture fixation using locking plates: device configuration and other considerations. Injury 49 Suppl 1: S12-S18.

Moazen M, Mak JH, Etchels LW, Jin Z, Wilcox RK, Jones AC, Tsiridis E (2013) The effect of fracture stability on the performance of locking plate fixation in periprosthetic femoral fractures. J Arthroplasty 28: 1589-1595.

Moazen M, Mak JH, Etchels LW, Jin Z, Wilcox RK, Jones AC, Tsiridis E (2014) Periprosthetic femoral fracture - a biomechanical comparison between Vancouver type B1 and B2 fixation methods. J Arthroplasty 29: 495-500.

Perren SM (2002) Evolution of the internal fixation of long bone fractures. The scientific basis of biological internal fixation: choosing a new balance between stability and biology. J Bone Joint Surg Br 84: 1093-1110.

Perren SM, Regazzoni P, Fernandez AA (2014) Biomechanical and biological aspects of defect treatment in fractures using helical plates. Acta Chir Orthop Traumatol Cech 81: 267-271.

Rodriguez EK, Zurakowski D, Herder L, Hall A, Walley KC, Weaver MJ, Appleton PT, Vrahas M (2016) Mechanical construct characteristics predisposing to non-union after locked lateral plating of distal femur fractures. J Orthop Trauma 30: 403-408.

Sanders R, Swiontkowski M, Rosen H, Helfet D (1991) Double-plating of comminuted, unstable fractures of the distal part of the femur. J Bone Joint Surg Am 73: 341-346.

Sommer C, Gautier E, Muller M, Helfet DL, Wagner M (2003) First clinical results of the locking compression plate (LCP). Injury 34 Suppl 2: B43-54.

Steinberg EL, Elis J, Steinberg Y, Salai M, Ben-Tov $\mathrm{T}$ (2017) A double-plating approach to distal femur fracture: a clinical study. Injury 48: 2260-2265.

Todorov D, Zderic I, Richards RG, Lenz M, Knobe M, Enchev D, Baltov A, Gueorguiev B, Stoffel K (2018) Is augmented LISS plating biomechanically advantageous over conventional LISS plating in unstable osteoporotic distal femoral fractures? J Orthop Res 36: 2604-2611.

Ziran BH, Rohde RH, Wharton AR (2002) Lateral and anterior plating of intra-articular distal femoral fractures treated via an anterior approach. Int Orthop 26: 370-373.

\section{Discussion with Reviewers}

Reviewer 1: With regards to the limitations of your study - how relevant are your findings for clinicians? Authors: Thank you for this comment. This study is very relevant for clinicians. Delayed union or nonunion in plate osteosynthesis is an important actual problem. In several cases, implant stability is the cause of nonunion and fracture healing can be achieved by an additional plate. The study demonstrates the enormous improvement in axial and torsional construct stability, compared to a single-plate fixation and gives therefore more insight in the already clinically applied helical-plating concept.

Reviewer 1: How can your study be improved to make it more relevant for clinicians?

Authors: Helical plating in a delayed fracture healing situation and as a primary osteosynthesis concept is one of the hot topics in actual orthopaedic trauma surgery. Although this concept has already been presented by Perren et al. (2014) in a similar plate configuration to the current study. The technique is nowadays widely applied. The authors do not feel that further improvement of the study is necessary, since axial and torsional loading are the typical load scenarios occurring in clinics.

Reviewer 1: What are the most crucial findings of your study and why?

Authors: Improved axial and torsional stiffness of the helical-plate constructs, especially in the gap situation, and reduced implant stress in the helical-plate configuration are obtained compared to single plating. These results are very relevant to the orthopaedic surgeon since delayed union and 
nonunion as well as implant failure is observed in single plating of unstable comminuted fractures.

Richard Stange: The distal part of the helical plate is attached to the femur over a long distance in your model. Which biomechanical differences would you expect in a more distal femur fracture situation?

Authors: Helical plating is a preferred technique in unstable fractures with lacking bony support, where lateral plating cannot act as tension banding and plate failure will occur. In more distally located fractures, the length of the helical plate could be shortened resulting in a stiffer construct.

Richard Stange: A relevant clinical problem is the need for distal medial plate fixation in metaphyseal trabecular bone, often compromised by osteoporosis in these fractures. Could you provide comments on that?

Authors: Indeed, osteoporotic metaphyseal fracture fixation is a challenging problem, which often requires a combination of two techniques. This might be lateral plating with an additional medial plate or, as proposed by Liporace and Yoon (2019), a retrograde nail with an additional lateral plate. These techniques have in common the provision of a well-balanced strengthened fixation construct, reducing implant stress by load sharing and resisting an increased loading compared to a single implant fixation.

Editor's note: The Guest Editor responsible for this paper was Anita Ignatius. 